Muséologies

Les cahiers d'études supérieures

muséologies

\title{
Les artistes contemporains au musée du Louvre : méthodes de transmission du processus créatif en regard des collections
}

\section{Ariane Lemieux}

Volume 8, numéro 1, 2015

Réflexions sur la pratique curatoriale et la recherche-création

URI : https://id.erudit.org/iderudit/1034616ar

DOI : https://doi.org/10.7202/1034616ar

Aller au sommaire du numéro

Éditeur(s)

Association Québécoise de Promotion des Recherches Étudiantes en

Muséologie (AQPREM)

ISSN

1718-5181 (imprimé)

1929-7815 (numérique)

Découvrir la revue

Citer ce compte rendu

Lemieux, A. (2015). Compte rendu de [Les artistes contemporains au musée du Louvre : méthodes de transmission du processus créatif en regard des

collections]. Muséologies, 8(1), 173-188. https://doi.org/10.7202/1034616ar

Tous droits réservés (C) Association Québécoise de Promotion des Recherches Étudiantes en Muséologie (AQPREM), 2016
Ce document est protégé par la loi sur le droit d'auteur. L'utilisation des services d'Érudit (y compris la reproduction) est assujettie à sa politique d'utilisation que vous pouvez consulter en ligne.

https://apropos.erudit.org/fr/usagers/politique-dutilisation/ 
Carnet deux

Les artistes contemporains au musée du Louvre: méthodes de transmission du processus créatif en regard des collections

Ariane Lemieux 
Ariane Lemieux est titulaire d'un doctorat en histoire de l'art de l'Université de Paris 1 Panthéon-Sorbonne. Sa thèse, L'artiste et l'art contemporain au musée du Louvre des origines à nos jours. Une histoire d'expositions, de décors et de programmations culturelles, propose une histoire de la transformation de l'institution en faveur du public depuis sa création en 1793 et du développement de ses politiques culturelles depuis la formation du Grand Louvre en 1983 à travers ses rapports avec les artistes et l'art contemporain. Ses recherches actuelles poursuivent cette réflexion et s'inscrivent dans le champ de celle sur l'évolution des rapports entre le musée d'art ancien et la création contemporaine. Elle est chargée d'enseignement à l'Université catholique de l'Ouest (Angers) et à l'Université de Paris 1 Panthéon-Sorbonne. ariane.lemieux.qc@gmail.com 
Entre 2004 et 2014, le musée du Louvre a pratiqué une politique d'ouverture à l'art contemporain en vue de stimuler chez les artistes d'aujourd'hui un travail issu d'une expérience des collections ou de l'espace dans lequel elles sont exposées en permanence. Cette politique se fondait sur le principe de la commande d'une œuvre nouvelle ou d'une remise à jour d'une œuvre existante et prévoyait son exposition temporaire en vis-à-vis de l'œuvre ancienne l'ayant inspirée. En initiant ce principe inédit, le musée du Louvre entendait redevenir, à l'image de ce qu'il fut au moment de son ouverture en 1793 et jusqu'à l'aube du $X X^{\mathrm{e}}$ siècle, un lieu de recherche et d'expérimentation pour les artistes et favorable à l'émergence de nouvelles problématiques ou thématiques. Les propos d'Henri Loyrette, président-directeur du Louvre de 2001 à 2013, sont révélateurs à maints égards: "L'objectif premier est de faire revenir les artistes car force est de constater que ces lieux ne constituent plus pour eux une source $d^{\prime}$ 'inspirations $»^{1}$. Favorisant une création qui témoigne d'une considération des collections que le Louvre ne souhaitait plus voir apparaître seulement comme le fruit des recherches en histoire de l'art, mais aussi comme une actualité vécue par les artistes du présent, les commandes en faveur de Jean-Michel Othoniel, Ange Leccia, Johan Creten ou encore lan Fabre, pour ne nommer que ceux-là, devaient ainsi modifier le regard des artistes sur le musée et sur ses collections. Mais elles devaient également modifier le regard du public. Si le Louvre voulait ouvrir le regard des artistes sur les collections, il voulait offrir au public un regard artistique sur les œuvres qu'il conserve et mettre celui-ci en présence de l'acte créatif dans la perspective d'une compréhension plus sensible des œuvres.

Les expositions des œuvres contemporaines réalisées par les artistes, sur invitation, $\mathrm{n}$ 'ont toutefois pas été conçues de manière à mettre en évidence leur recherche et leur réflexion sur les œuvres du musée ou leur espace d'exposition, à l'exception peut-être de celles de William Kentridge en 2010 et Bob Wilson en 2013. Il s'agissait dans ces deux cas particuliers d'une pratique caractéristique de leur œuvre. En revanche, à défaut d'une mise en exposition de la recherche de l'artiste ou de son processus créatif menant à l'œuvre finale pour le Louvre, des moyens ont été mis en place pour que le public puisse prendre acte, dont la publication d'entretiens entre la commissaire chargée de l'art contemporain et les artistes, et l'organisation de rencontres entre les artistes et le public, de conférences et de projections documentaires sur l'œuvre de l'artiste invité.

\section{Témoigner du rôle des collections du Louvre dans le processus créatif des artistes}

Cette politique de la commande spécifique trouve sa genèse dans la réflexion plus générale sur la nécessité pour le Louvre d'ouvrir sa programmation à la création contemporaine et de confronter ses 
collections à celle-ci. En 1981, l'annexion, au musée, de l'aile Richelieu a été l'occasion de plusieurs changements qui ne se sont pas limités à l'augmentation de la surface d'exposition et à la modernisation des conditions d'accueil et de présentation des œuvres. Le Grand Louvre, terme utilisé pour signifier l'extension du musée sur l'ensemble du Palais, devait devenir un ensemble culturel dans lequel le public pouvait assister à des conférences, des productions audiovisuelles et interactives, mais aussi, bien évidemment, où il pouvait visiter d'importantes expositions temporaires aux discours thématiques ou monographiques. Si cette transformation du Louvre devait se manifester par la mise à jour de ses installations et de son organisation, elle devait aussi se concrétiser par une ouverture plus importante à la diversité des expressions artistiques dans le cadre d'une programmation culturelle soutenue par la création d'une importante salle d'expositions temporaires - dont le Louvre ne disposait toujours pas à l'époque ${ }^{2}$ - et d'un auditorium.

Cette ouverture envisagée du musée à la création contemporaine a immédiatement suscité des propositions qui, selon leurs auteurs, favorisaient soit l'exposition, soit la production. Parmi elles, celle du directeur de la toute nouvelle Délégation des arts plastiques (DAP) chargée de favoriser et de promouvoir la création contemporaine, qui consistait à présenter au Louvre une exposition annuelle d'art contemporain, comme au temps des Salons ${ }^{3}$, et à ouvrir des ateliers au profit $d^{\prime}$ artistes-boursiers de tous les pays. Cette proposition, qui s'inspirait de l'histoire du musée du Louvre, semblait d'autant plus opportune aux yeux du délégué aux arts plastiques, Claude Mollard, que le ministre de la Culture, Jack Lang, prônait une ouverture du patrimoine " au bouillonnement du temps présent ${ }^{4}$. Certes, il régnait alors, comme l'explique Michel Laclotte, président-directeur du musée de 1987 à 1995 , un sentiment selon lequel «le musée ne pouvait plus demeurer simplement un conservatoire du patrimoine et un centre de recherche scientifique, mais qu'il devait devenir aussi un lieu d'accueil, accessible à toutes sortes de publics nouveaux ou négligés, et éventuellement un lieu de création, ouvert à l'art contemporain $»^{5}$.

2 Il est vrai que la Galerie Mollien, accessible par l'ancienne entrée du musée qui se faisait à l'époque par la porte Denon, existait depuis 1960. Bien que localisée dans le musée du Louvre, cette petite galerie d'expositions temporaires était placée sous la responsabilité de la direction des musées, donc du ministère de la Culture, et était par conséquent à la disposition de tous les conservateurs des musées nationaux. Elle présentait donc autant d'expositions temporaires du musée du Louvre que du Musée national d'art moderne ou du musée Guimet consacré aux arts asiatiques.

3 De l'ouverture du musée du Louvre en 1793 jusqu'à la Révolution de 1848, les salons de la peinture et de la sculpture étaient organisés dans le Salon carré, où étaient exposés les plus grands chefs-d'œuvre de la collection de peinture. L'usage du Salon carré pour l'exposition biannuelle de l'art contemporain de l'époque cessa définitivement avec le Salon de 1848 , pour trois motifs: le non-sens du nécessaire retrait des chefs-d'œuvre anciens pendant plusieurs mois au profit des œuvres contemporaines; l'augmentation constante du nombre d'œuvres à exposer obligeant d'étendre aux salles adjacentes au Salon Carré; l'évolution même de la création contemporaine. Voir, à ce sujet, CHAUDONNERET, Marie-Claude. Le Salon pendant la première moitié du XIXe siècle. Musée d'art vivant ou marché de l'art?, 2007. <halshs-00176804> (consulté le $1^{\text {er }}$ septembre 2014).

4 Jack Lang lors d'un discours devant l'Assemblée nationale le 17 janvier 1981, repris dans MOLLARD, Claude. Le Mythe de Babel. L'Artiste et le système. Paris: Bernard Grasset, 1984, p. 74 .

5 LACLOTTE, Michel. Histoires de musées. Souvenirs d'un conservateur. Paris: Scala, 2003, p. 244. 
Il demeurait néanmoins difficile de concevoir un projet favorable à la création et à la recherche des artistes vivants. L'agrandissement et la modernisation du Louvre impliquaient d'importants travaux d'aménagement, la mise en place d'une nouvelle organisation du travail, la création de nouveaux services, condition sine qua non d'une institution moderne ouverte sur l'avenir, et l'invention d'une politique culturelle et d'un programme d'expositions temporaires capables de stimuler I'intérêt du public de proximité envers les collections. Dans ces conditions, la direction et les conservateurs considéraient que l'ouverture à l'art contemporain ne devait pas prendre la tangente proposée par le délégué aux arts plastiques qui, au sortir de plusieurs années de grands travaux, aurait brouillé l'identité même de l'institution. De leur avis, il valait mieux limiter cette ouverture, du moins dans un premier temps, aux expositions temporaires. Ils défendaient ainsi davantage un programme d'expositions thématiques qui associeraient les œuvres du musée à celles d'artistes modernes et contemporains les ayant largement considérées dans le cadre de leur recherche technique ou créatrice.

Il est vrai qu'à l'occasion de leur travail sur la programmation future du musée, mais aussi des activités devant marquer le bicentenaire du Louvre en août 1993, les conservateurs ont fait un état des lieux de l'histoire du musée qui encourageait cette idée de confrontation ancien-contemporain. L'ouverture à l'art contemporain pouvait donc se réaliser dans une perspective historique par une mise en lumière des rapports qu'entretenaient les artistes d'aujourd'hui avec les maîtres des siècles passés. L'exposition Copier-créer. De Turner à Picasso: trois cents œuvres inspirées par les maîtres du Louvre, présentée au Louvre en 1993, est certainement la plus emblématique de toutes celles qui ont été réalisées dans cet esprit ${ }^{6}$. En confrontant les œuvres les plus célèbres du Louvre à leurs copies libres, celles où «I'artiste met sa marque par la simple rapidité de son geste ou en interprétant l'original parfois en le transformant considérablement ${ }^{7}$, l'exposition démontrait que le processus créatif $d^{\prime}$ artistes des $X I X^{e}$ et $X X^{e}$ siècles s'appuyait largement sur les chefs-d'œuvre du Louvre. Elle offrait une histoire des collections du Louvre à partir d'une mise en lumière du processus artistique d'une pluralité d'artistes allant de la fin du XVIIe siècle à la fin du $X X^{e}$ siècle. "Est-il légitime d'envisager, écrivait le commissaire Jean-Pierre Cuzin, une histoire de l'art - disons ici de la peinture, et plus particulièrement de la peinture française réduite à deux siècles qui aborderait en termes stylistiques le rapport que les artistes entretiennent envers leurs modèles et la façon dont ils les ont intégrés, assimilés, exploités (ou simplement cités, ou rejetés) ${ }^{8}$ ?"

6 Notons Polyptyques: le tableau multiple du Moyen Âge au XXe siècle (1990), Égyptomania. L'Égypte dans l'art occidental. 1730-1930 (1994) et D'Après l'Antique (2000).
7 CUZIN, Jean-Pierre. Copier-Créer. De Turner à Picasso: trois cents œuvres inspirées par les maîtres du Louvre, Musée du Louvre, Hall Napoléon. Paris: Réunion des musées nationaux, 1993, p. 27.

8 Id., p. 30. 
Ouvrant sur un nouveau champ d'investigation quant à l'histoire des œuvres du musée, cette réflexion permettait un large éventail de sujets d'expositions qui, non seulement assurait une ouverture à des œuvres qui ne s'inscrivaient pas dans les limites temporelles définies par le mandat du Louvre, mais aussi réintégrait les œuvres des collections dans une histoire vivante de l'art. En outre, en démontrant que le musée du Louvre avait eu pour vocation de soutenir la formation des artistes et qu'il était toujours un lieu d'idées et de réflexion pour eux, le programme d'expositions sur les rapports anciens-modernes encourageait l'idée d'une politique qui favoriserait la poursuite d'un usage des collections par les artistes vivants. Le principe de la commande se posait alors comme une évidence. En effet, dès lors qu'il s'agissait de démontrer l'apport des œuvres du Louvre dans le processus créatif des artistes contemporains, la confrontation des œuvres anciennes et des travaux préparatoires que représentent les copies libres perdait en faisabilité et, surtout, en pertinence. Non seulement la copie ne fonctionne plus comme processus créatif, mais les tableaux de maîtres ne sont plus des objets de recherche privilégiés, si ce n'est dans un registre postmoderniste qui se caractérise par un usage des références historiques selon un angle critique et/ou ironique. Qui plus est, il s'agissait de démonstrations qui se concentraient sur des œuvres déjà réalisées et qui, finalement, étaient soumises à un discours qui visait d'abord la mise en valeur des collections. Devant une création détachée de la valeur d'exempla des œuvres anciennes et ne voulant pas se limiter à une mise en avant des phénomènes de revivals, de détournement, de transpositions ou de citations, visant le retour des artistes et l'intégration de leur regard sur les collections, le musée du Louvre a donc pris le parti d'inviter les artistes à revisiter leurs problématiques et à réengager leur processus créatif à la lumière d'une ou de plusieurs des œuvres exposées en permanence.

\section{Stimuler un processus créatif en regard de l'art ancien}

L'apparition d'un programme pour favoriser la création en art contemporain est le fait de l'arrivée d'Henri Loyrette à la tête du musée du Louvre en 2001. II n'aura pas fallu longtemps avant que ce conservateur général du patrimoine, ancien directeur du musée d'Orsay, s'y attèle. En novembre 2002, il signait une convention d'une durée de trois ans avec le Centre national des arts plastique (CNAP) afin de " susciter des rencontres entre la création contemporaine et l'art du passé, d'organiser des dialogues croisés entre des formes d'expression diversifiées et de favoriser l'élaboration de points de vue singuliers d'artistes contemporains sur des espaces/temps artistiques antérieurs $»^{9}$. L'objectif visé était spécifiquement de faire participer l'artiste à l'intelligence des œuvres du passé et de contribuer à son exploration créatrice en l'engageant sur de nouveaux axes de recherche à partir d'un corpus d'œuvres anciennes 
rassemblées dans une exposition temporaire. James Coleman, artiste irlandais reconnu pour ses installations et ses vidéos aux ambiguïtés narratives, est cependant le seul à avoir bénéficié de cette convention inédite. Invité dans le cadre de l'exposition Léonard de Vinci: dessins et manuscrits présentée au Louvre en 2003, Coleman devait développer un projet prenant appui sur sa réflexion sur l'œuvre graphique et manuscrite du maître italien et sur La Dernière Cène. Selon la note d'intention de la commissaire, Françoise Viatte, il lui a été demandé de « recréer, de façon très libre, le cheminement mental du maître italien et témoigner de l'importance de son travail préparatoire à travers l'évocation de son œuvre peinte et dessinée $»^{10}$. Le processus créatif était alors à la fois le sujet de la proposition de l'artiste et l'objet de l'invitation.

Dans le but de faire comprendre au public la manière dont l'artiste avait abordé intellectuellement la commande du Louvre et du CNAP, il était prévu d'éditer en parallèle une publication spécifique avec un texte de Daniel Arasse, en tant que spécialiste de Léonard de Vinci, mais aussi fin connaisseur de l'art contemporain: « La commande de ce texte vient de Marion Sauvaire et de Jean-Loup Champion et devait inaugurer une collection coproduite par la délégation aux Arts plastiques, le musée du Louvre et Gallimard ${ }^{11}$. " Or, l'artiste n'a jamais accepté le texte dans lequel l'auteur l'abordait « son rapport à l'œuvre de Léonard, faisant voir comment il [l'artiste] nous fait voir l'art et ce qu'il fait voir ". II n'a pas davantage accepté de rendre une documentation sur son projet, comme le requérait son contrat avec le musée du Louvre et le CNAP. En effet, en vue d'assurer une compréhension et la diffusion du processus créatif particulier que pouvait générer ce type de commandes, il était prévu que les éléments de la recherche de l'artiste ainsi que ses travaux préparatoires soient déposés aux archives du CNAP. Cette documentation, comme le précisait la convention, devait constituer en elle-même " une œuvre réalisée spécifiquement pour le CNAP en un exemplaire unique $"{ }^{12} \mathrm{Et}$ de continuer que ce modèle de documentation inclurait, le cas échéant, « une sélection d'images et de dessins d'études effectué par l'artiste, en accord avec le CNAP "13, et serait inscrit aux inventaires du Fond national d'art contemporain (FNAC). Cela impliquait d'éventuels prêts ou mises en dépôt dans les conditions habituelles régissant le fonctionnement du FNAC. La convention soutenait donc un programme de diffusion du processus créatif de l'artiste bénéficiant d'une commande qui lui demande de développer un regard et une réflexion sur un corpus spécifique d'œuvres anciennes.

Au terme des trois ans de la convention, la décision de ne pas la renouveler découlait d'une analyse approfondie. L'expérience avec Coleman avait révélé que le sujet de l'exposition temporaire rendait invisible sa proposition. La fatigue physique et cérébrale provoquée par la visite

10 VIATTE, Françoise. Note d'intention sur une installation audiovisuelle dans l'exposition "Léonard de Vinci; dessins et manuscrits ", Dossier James Coleman, Archives de la DAP.
11 BÉDARD, Catherine. "Préface». In. ARASSE, Daniel et Catherine BÉDARD, Anachroniques. Paris: Gallimard, 2006, p. 11.

12 Ibid.

13 Contrat de commande d'œuvre, 18 novembre 2002. Archives de la DAP. Dossier James Coleman. 
d'une exposition temporaire au dispositif narratif soutenu était, semblet-il, peu favorable à la lecture et à l'appréciation d'une œuvre qui était en décalage stylistiquement et historiquement. La direction du Louvre souhaitait néanmoins poursuivre son action concernant la création contemporaine et la recherche en présentant aux artistes les collections permanentes comme l'objet de départ d'un nouveau projet créatif.

Annoncée en octobre 2003, l'adoption d'une politique interne offrant aux artistes la possibilité de renouveler leur questionnement et leur pratique à partir des collections permanentes a permis l'organisation d'une trentaine d'expositions d'art contemporain dans le parcours de visite sur un peu plus de dix ans. Celles-ci se sont concrétisées grâce à plusieurs types d'invitations qui, entre 2004 et 2014, ont connu des évolutions diverses pour des raisons logistiques ou des circonstances momentanées. Le premier type d'invitation s'adressait simultanément à une dizaine d'artistes à qui il était demandé d'intervenir dans les collections permanentes, soit en produisant une œuvre spécifique, soit en montrant une œuvre déjà existante, soit encore en reconstituant une œuvre spécialement adaptée à l'espace de présentation. Il en a résulté trois expositions collectives qui avaient pour spécificité de placer en vis-à-vis les œuvres réalisées par les artistes invités et les œuvres anciennes à partir desquelles ils avaient choisi de travailler: Contrepoint. L'Art contemporain au Louvre (2004) avait pour spécificité d'investir tout le musée; Contrepoint au Louvre. De l'objet d'art à la sculpture. Porcelaines contemporaines (2005) se concentrait dans le département des Objets d'art; puis Contrepoint au Louvre. De la sculpture (2007) se déployait dans le département des Sculptures et celui des Antiquités orientales. À partir de 2008, le Louvre prenait le parti de n'inviter qu'un seul artiste à la fois. Dans le département de son choix, celui-ci pouvait soit intégrer ses œuvres existantes, soit présenter une œuvre spécialement réalisée pour le Louvre, soit les deux. Un autre type d'invitation consistait à demander à des artistes de réaliser une exposition de type installation qui témoignerait de leur rapport au patrimoine artistique. II s'agissait alors pour eux d'ouvrir leurs problématiques habituelles à une réflexion sur l'apport encore possible de l'art ancien en ce début du troisième millénaire. Des artistes usant du médium de la photographie étaient, pour leur part, invités à réaliser un projet qui conduirait les visiteurs à découvrir le musée et ses collections sous un angle à la fois documentaire et poétique. Certains, en raison de la forme et de la nature du travail sur les collections et de l'approche qu'ils en faisaient, ont bénéficié d'un atelier dans le Louvre. Enfin, des occasions particulières pouvaient également stimuler de nouveaux types d'invitations qu'il n'est pas utile ici de relever. II importe peut-être seulement de souligner que la diversité des invitations et des formes d'expositions visait à témoigner de la volonté du Louvre d'offrir aux artistes des perspectives de recherches nouvelles dans un contexte souple mais néanmoins surdéterminé. 


\section{La diffusion des processus créatifs engagés par les invitations du Louvre}

L'objectif du programme du Louvre étant, tout à la fois, la réactualisation de ses collections à travers la recherche créatrice d'un artiste et la démonstration de la pérennité des dialogues entre art contemporain et art ancien, il n'était pas opportun qu'il se limite à une simple exposition des œuvres, inédites ou non, de ses glorieux invités. Pour gagner en pertinence, le programme devait témoigner du cheminement intellectuel de leur projet et des idées qu'ils avaient dégagées de leur confrontation aux œuvres anciennes. Plusieurs voies ont été ouvertes en ce sens. Des rencontres entre le public et les artistes invités ont été organisées dès le départ, selon des modalités diverses mais qui devaient toutes mettre en évidence le cheminement mental provoqué par les commandes. Parmi elles, notons celle qui faisait de l'artiste le médiateur de sa réflexion afin d'établir un dialogue direct entre son œuvre et le public. L'apparition de l'« artiste-médiateur » au Louvre est incontestablement associée à sa volonté d'une ouverture à l'art contemporain.

Déjà, en 1993, l'équipe du service culturel, dirigée à l'époque par Jean Galard, avait appuyé le propos de l'exposition Copier-créer. De Turner à Picasso: trois cents œuvres inspirées par les maîtres du Louvre en invitant des artistes à venir parler de l'apport d'une œuvre des collections sur leur propre travail et réflexion. Par le biais de ces invitations, inspirées des pratiques des musées d'art contemporain et en particulier du Musée d'art moderne de la ville de Paris ${ }^{14}$, il s'agissait alors d'intégrer, dans l'enceinte du Louvre, un discours verbal et instantané sur la pratique artistique contemporaine et la recherche qui $s^{\prime} y$ associe. Intitulé Propos d'artiste, ce programme visait les étudiants en arts des universités de Paris I, Paris III, Paris IV et Paris VIII que I'on voulait encourager à considérer les collections du Louvre dans le cadre leur recherche. "Le Louvre, expliquait la responsable de ce programme particulier du service culturel, s'il est certes un lieu de conservation des œuvres, est aussi un lieu vivant. Car même si l'image du Musée a changé, il reste encore pour trop d'étudiants un temple qu'ils n'osent pas fréquenter, et les artistes du passé ont pour eux valeur de patrimoine, et sont totalement désincarnés ${ }^{15}$. "

14 En 1967, la création du projet " Animation, recherche, confrontation " (l'A.R.C.), confié à Pierre Gaudibert, conservateur adjoint du Musée d'art moderne de la ville de Paris, visait " un retour aux sources du musée, lieu de travail et de formation des artistes, dans un temps de crise de l'enseignement artistique, recherche de nouveau public ". (MONNIER, Gérard. L'Art et ses institutions en France. De la Révolution à nos jours. Paris: Gallimard, p. 358). Dans ce contexte, caractérisé par une série d'initiatives afin que le musée n'apparaisse plus comme " un entrepôt d'objets, un espace de contemplation et de rêverie solitaire, mais aussi comme un agent du changement artistique et un espace de réflexion sur l'art en général ", Gaudibert décidait de faire appel aux artistes pour s'assurer d'une " nouvelle approche des arts par l'actualité " et d'un « regard critique sur les arts du passé ». Par la " présence animatrice " de l'artiste, il s'agissait également de susciter de nouvelles relations entre les publics et les aventures artistiques d'aujourd'hui et de "promouvoir une pédagogie active de sensibilisation à l'art contemporain ». La présence de l'artiste se traduisait alors par sa participation à des conférences, à des visites guidées ou à des discussions autour de ses œuvres, et d'autres, exposées en permanence au Musée d'art moderne de la ville de Paris. Voir, à ce sujet, GAUDIBERT, Pierre. "Problèmes du musée d'art contemporaine en Occident ". In. DESVALLÉES, André (dir.) Vagues, Une anthologie de la nouvelle muséologie. Mâcon: Éditions W, 1992, tome II p. 145-179.

15 TUFFELI, Nicole. «Propos d'artiste au Musée du Louvre ". Musées et Collections publiques en France, $\mathrm{n}^{\circ} 200$, septembre 1993, p. 15. 
Le service culturel a invité au total quatre artistes: Ernest Pignon-Ernest, François Morellet, Gérard Titus-Carmel et Pierre Buraglio. Ce ne sont cependant que les deux premiers qui ont tenu des propos révélateurs de leur processus artistique. Titus-Carmel et Buraglio s'étaient en effet davantage efforcés de partager leur interprétation iconologique et esthétique des œuvres qu'ils avaient choisies. À l'inverse, Pignon-Ernest s'était attaché à expliquer pourquoi il avait travaillé à partir de La Mort de la vierge de Caravage pour répondre à une commande publique de la Ville de Naples et comment ce travail s'était formulé peu à peu. Les auditeurs percevaient ainsi tout son processus de réflexion et les étapes du travail qui en a résulté. Devant La Bataille de San Romano de Paolo Uccelo, Morellet a d'abord traité de l'écho qu'il y voyait dans son propre travail caractérisé par un jeu d'obliques et de parallèles. Dans un deuxième temps, il donnait à comprendre comment l'observation et l'analyse formelle d'une œuvre ancienne nourrissaient ses propres recherches et réflexions en vue de réaliser ses œuvres.

Dans la perspective d'une mise en lumière des œuvres contemporaines exposées, la politique d'ouverture des années 2004-2014 appelait cette présence animatrice de l'artiste au Louvre. Des rencontres in situ, c'est-à-dire face à l'œuvre réalisée, ont ainsi été organisées pour permettre aux artistes d'expliquer, dans leurs mots, la manière dont ils avaient abordé la commande du Louvre. Destinées aux jeunes de 18 à 30 ans, ces rencontres devaient ainsi mettre en évidence le pourquoi de la forme de l'œuvre présentée et le cheminement mental qu'elle reflétait. La première rencontre a été organisée lors de l'exposition À côté rêve un sphinx accroupi, des photographes au Louvre, atelier de Patrick Faigenbaum, présentée du 12 novembre 2004 au 10 janvier 2005.

Cette exposition, qui résultait de l'invitation du photographe et de ses étudiants de l'École de beaux-arts à la réalisation d'un projet photographique, voulait suggérer « un point de vue singulier et kaléidoscopique sur le Louvre ". Lors de leur rencontre avec le public, les jeunes artistes ont établi un dialogue pour le moins fructueux qui a su démontrer comment ils avaient abordé le projet, comment ils avaient choisi les lieux, combien de temps ils avaient pris pour regarder, pourquoi ils avaient choisi de faire des études et des esquisses avant de photographier, qu'est-ce qu'ils avaient observé, pourquoi, dans quel esprit.

La même année, une nouvelle rencontre était organisée avec les artistes de la première exposition dans les salles réservées aux collections permanentes: Contrepoint. L'art contemporain au Louvre. Cette fois, le service culturel organisa des rencontres non seulement avec les artistes, mais aussi avec Marie-Laure Bernadac, la chargée de l'art contemporain au Louvre et commissaire de l'exposition, et des conférenciers. Ces interventions multiples et simultanées devaient certes assurer une médiation sur le pourquoi d'une telle exposition dans le 
parcours des collections permanentes que certains, voire plusieurs, pouvaient trouver incongrue. Mais elles avaient pour principal objectif de faire comprendre aux visiteurs la diversité des problématiques que les artistes avaient abordées lors de leur invitation et la particularité du processus créatif devant répondre à une commande qui exigeait l'élaboration de points de vue singuliers sur les collections et leur espace de présentation. En clair, ces interventions médiatrices devaient démontrer comment les œuvres anciennes pouvaient participer au développement de la réflexion et à l'avancement de la recherche-création auprès des artistes d'aujourd'hui.

En 2007, Sarkis, habitué du travail en contexte muséal, avait été invité à réaliser une installation qui témoignerait de ses dialogues avec l'art ancien tout au long de sa carrière. Le service culturel lui avait demandé d'échanger avec le public, devant son œuvre, sur le développement de ses réflexions et de ses recherches dans le contexte de son projet pour le Louvre. Lors de son intervention, il a ainsi donné les raisons de la rencontre qu'il avait imaginée entre le Cri de Munch, le Retable d'Issenheim de Grünewald, La Bataille de San Romano de Paul Uccello et le Werkkomplex de Joseph Beuys. II a expliqué les problématiques qu'il abordait de manière continue de façon à ce que le public présent puisse appréhender la nature physique et psychique de sa proposition pour le Louvre. Son intervention apportait ainsi un l'éclairage pertinent sur le cheminement de sa réflexion à partir de la réception de son invitation et sur comment il en était arrivé à l'idée de conceptualiser et de matérialiser, à l'aide des nouvelles technologies, la rencontre des quatre chef-œuvres qui ont eu une incidence si particulière sur la formation de sa pensée artistique.

De nouvelles rencontres étaient également organisées à la faveur de l'exposition Contrepoint: de la Sculpture (2007) pour laquelle une dizaine d'artistes avait été invités à sélectionner, de manière individuelle, une œuvre ou un espace spécifique dans le département des Sculptures que le Louvre avait voulu mettre en valeur. Cette fois, le service culturel organisa des visites avec des médiateurs spécialisés tous les samedis que dura l'exposition afin d'expliquer les œuvres contemporaines exposées. Il organisa également une rencontre in situ avec une des artistes invités, Élisabeth Ballet ${ }^{16}$. Sa sculpture en résine représentant un preneur de son « en action » était insérée au milieu d'un groupe de sculptures du XVII siècle exposées dans la cour Marly qu'elle avait replacées de façon à ce qu'elles semblent discuter entre elles. Lors de la rencontre avec le public, I'artiste a expliqué, pour commencer, les problématiques auxquelles elle s'attachait habituellement dans son travail. Après cette mise en contexte, elle a partagé le cheminement de ses réflexions jusqu'à l'idée de son Perchman. L'un des points forts de son intervention a certainement été la révélation des effets du contexte du Louvre sur son acte créateur. Elle a expliqué le besoin qu'elle avait ressenti de revoir ses pratiques habituelles, prétex- 
tant pour l'essentiel qu'elle avait été incapable de visualiser son travail de nature abstraite à côté des sculptures exposées dans la cour Marly. Par ses propos, le public prenait réellement conscience des différentes strates de questionnements qu'impose une commande comme celle du Louvre et des effets du contexte sur les choix technique et plastique.

L'intégration de la parole de l'artiste dans le musée est assurément I'un des moyens les plus pertinents pour assurer une transmission de la réflexion sous-jacente à la réalisation de son œuvre. Elle permet un dialogue direct avec le public dont les questions visent généralement à comprendre le processus créatif qui est nécessairement aléatoire et assurément difficile à concevoir par le simple regard. Or, cette parole ne bénéficie qu'à un nombre limité de visiteurs et elle est, de surcroît, confrontée à l'oubli. Pour conserver une trace de l'expérience des artistes, le Louvre éditait un numéro spécial en partenariat avec le magazine Beaux Arts ou publiait un catalogue dont les textes devaient rendre intelligible la recherche des artistes qui relevaient le défi de la commande spécifique en contexte muséal.

Comme le rappelait Didier Rykner, dans son éditorial pour la Tribune de l'Art, le catalogue d'exposition n'est pas un ouvrage comme les autres, il a un rôle spécifique qui consiste à traduire, par le verbe et en images, un rassemblement d'œuvres ou la réalisation d'un projet artistique inédit à l'initiative d'un musée ou d'une galerie ${ }^{17}$. Il apporte des informations pertinentes sur les œuvres mises en exposition et sur les motifs de cette dernière. Si l'œuvre créée par l'artiste demeure, son contexte de présentation, qui est constitutif de sa forme et de son sens, est éphémère. Mémoire d'un donné à voir et à comprendre à un moment précis, le catalogue se révèle donc un outil fondamental de transmission des connaissances, mais aussi de partage des points de vue et du processus créatif des œuvres présentées. II participe en ce sens à la recherche historienne et critique qui, d'elle-même, œuvre en faveur d'une diffusion, dans la longue durée, de la recherche créative de l'artiste et de son œuvre. Le Louvre a vite compris que l'exposition temporaire d'œuvres contemporaines issues de son programme de commandes auprès d'artistes de renom nécessitait une publication particulière. C'est par ce biais qu'il s'assurait de faire comprendre aux visiteurs le contexte de production de l'œuvre - en l'occurrence une invitation à une confrontation - et le cheminement mental de l'artiste.

Les catalogues s'organisaient autour d'un entretien entre la commissaire et l'artiste. Le choix de l'entretien est révélateur d'une volonté de transmettre le processus créatif qui a précédé l'œuvre. L'entretien est une forme ancienne de discours utilisée par de nombreux historiens d'art pour faire comprendre comment l'œuvre de l'artiste s'est constituée. Sa pratique remonte au XVII e siècle et trouve ses premières expressions dans les dix Entretiens sur les vies et sur les ouvrages des 
plus excellents peintres anciens et modernes d'André Félibien ${ }^{18}$. Bien que fictifs, ils avaient été conçus pour faciliter la compréhension des préceptes théoriques et des informations " techniques » et ainsi donner des éléments d'analyse pertinents sur le travail d'un artiste. Dans la seconde moitié du XX' siècle, l'entretien a pris une nouvelle importance avec l'affirmation d'un art s'appuyant sur une théorie philosophique et une réflexion conceptuelle au détriment de l'esthétique et du savoirfaire de la main. Comme l'ont démontré les interventions lors du colloque Paroles d'artistes, organisé par le Centre Pompidou en avril 2013, c'est largement par le biais de questions sur le quoi, le comment et le pourquoi que sont aujourd'hui retirés les éléments d'analyse pertinents du travail de l'artiste ${ }^{19}$.

Dans le contexte des invitations du Louvre, ce jeu de questions-réponses entre les artistes et la chargée de l'art contemporain allait dans le même sens: comprendre et mettre en évidence la réflexion de l'artiste et un processus créatif déterminé, en partie, par le cadre spécifique de la commande d'un musée comme le Louvre. Marie-Laure Bernadac cherchait ainsi à connaître la relation de l'artiste au musée de manière générale, puis à discerner le cheminement de ses idées constitutives à la proposition du Louvre. Les invitations du musée se voulant génératrices d'une réflexion spécifique sur l'art ancien et porteuses de nouveaux axes de recherche, l'entretien visait par ailleurs à découvrir comment, à travers sa réalisation, l'artiste avait poursuivi et enrichi ses problématiques habituelles. Yan Pei Ming, par exemple, répondait à des questions sur le sens de son projet sur La Joconde qu'il dit avoir choisi dès la réception de son invitation. Wim Delvoye reprenait la genèse de son projet au Louvre, ses premières idées, ses rêves et sa relation au musée. Il lui était d'ailleurs demandé d'expliquer comment ce projet pour le Louvre poursuivait son travail sur les tours gothiques. Joseph Kosuth expliquait, pour sa part, comment et pourquoi il avait choisi les remparts médiévaux pour la réalisation de son installation lumineuse, ainsi que le sens qu'elle revêtait. Bref, à travers le principe de l'entretien, il s'agissait d'apporter un éclairage constructif sur la réflexion engagée par les artistes à l'occasion de leur invitation au Louvre.

L'entretien entre Bernadac et les artistes était systématiquement suivi d'un essai dont le but était d'apporter une perspective plus intellectualisée de l'œuvre. Rédigé par un spécialiste externe au musée, il complétait les propos tenus par l'artiste sur la façon dont il avait abordé son travail au Louvre. Offrant une analyse des problématiques soulevées par l'ensemble des œuvres produites par l'artiste, l'essai participait à une meilleure compréhension de la pratique de ce dernier et donc de ses choix pour le Louvre. Le contenu du catalogue proposait ainsi une articulation entre pratique et théorie utile à une pénétration plus riche des recherches de l'artiste et de la nature de son travail. Cette articu-

18 FÉLIBIEN, André. Entretiens sur les vies et sur les ouvrages des plus excellents peintres anciens et modernes. Paris: Les Belles Lettres, 1986. Deux volumes présentés par René Démoris.
19 Colloque international Paroles d'artistes. Paris: Centre Pompidou, 11-12 avril 2013. Le colloque est en ligne: <http:// www.dailymotion.com/video/x10ef3y_paroles-d-artistescolloque-international-le-11-avril-2013_creation> (consulté le 15 septembre 2014). 
lation était également proposée lors des conversations devant public entre l'artiste et l'auteur de l'essai ou un autre spécialiste (historien, critique, philosophe, praticien travaillant des problématiques similaires de manière différente). Mises en place par l'équipe de l'auditorium, ces conversations devaient ouvrir la réflexion et le débat autour de l'art contemporain en prenant pour point de départ l'œuvre de l'artiste invité et plus spécifiquement, parfois, celle qu'il a créée pour le Louvre. Ainsi, si l'entretien du catalogue avait comme objectif d'expliquer la démarche de l'artiste issue de son invitation, la conservation publique visait, pour sa part, une exploration plus large des éléments constitutifs de la pratique globale de l'artiste. Cependant, toutes les conversations entre artistes et spécialistes, intitulées Faces-à-Faces, n'ont pas toujours offert des informations claires sur le processus créatif de l'artiste.

Certaines prenaient des axes si conceptuels qu'il était difficile pour le public de le discerner clairement. Mais, de manière générale, le principe des Faces-à-Faces était une avenue intéressante pour transmettre le fonctionnement de l'artiste et mettre en lumière différentes réalités et logiques de la création (matérielles, relationnelles, contextuelles, psychiques...).

Lorsque le matériel le permettait, l'auditorium organisait également des projections de documentaires. Celle organisée lors de l'invitation de Michelangelo Pistoletto est exemplaire à maints égards. Elle offrait en continu, dans la salle audiovisuelle du hall d'accueil, deux courts documentaires permettant de mieux cerner la pratique de l'artiste et ses axes de recherches, suivis de trois courts films donnant à voir l'une de ses nombreuses performances. Même si le projet spécifique pour le Louvre ne faisait l'objet d'aucun commentaire, le contenu des projections contribuait à sa compréhension. Toujours dans le dessein de faire comprendre la spécificité de l'œuvre réalisée pour le Louvre, mais aussi la démarche même du musée, notons l'organisation d'un colloque international à l'occasion de l'invitation de Jan Fabre. En présence de l'artiste et autour de documents d'archives, de films anciens, récents ou inédits, les intervenants abordaient les différentes problématiques de son œuvre et le sens de son travail. Son exposition pour le Louvre, "L'Ange de la métamorphose ": Jan Fabre et la peinture ancienne, faisait l'objet d'une contribution à part entière. Bref, de nombreux exemples pourraient être cités ici. Cependant, ce qui est important de retenir, c'est que la diffusion des processus-créatifs de l'artiste et des réflexions qui sous-tendent son œuvre doit prendre en compte tous les services du musée et s'inscrire dans la programmation culturelle globale de l'institution.

\section{**}

L'ouverture du Louvre aux artistes avait pour but de redonner aux collections le sens de leur existence artistique tout en montrant aux visiteurs qu'une œuvre d'art, quelle que soit l'époque à laquelle elle appartient, sous-tend une réflexion individuelle, procède d'une recherche complexe et dépend d'un processus créatif spécifique. 
"C'est pour montrer qu'un musée est le résultat d'œuvres réalisées par des artistes qui ont été vivants et contemporains. Les œuvres sont le fruit d'un parcours personnel, d'une démarche créatrice, d'une vie, même si elles se retrouvent dans un musée ", expliquait en ce sens Marie-Laure Bernadac ${ }^{20}$. Durant les dix années de cette politique inédite en faveur de l'art contemporain, le musée du Louvre a stimulé des œuvres et produit des expositions révélatrices, de façon plus ou moins évidente selon les cas, de la réflexion d'un artiste sur l'histoire, la nature, ou le sens d'une ou de plusieurs œuvres exposées en permanence. Servant à offrir une autre vision des collections, la diffusion de cette réflexion ne pouvait être négligée ou laissée de côté. En effet, si les œuvres réalisées soutiennent un point de vue singulier de l'artiste sur une œuvre des collections ou encore l'espace dans lequel elles sont présentées, celui-ci est difficilement déchiffrable. La parole de l'artiste, qu'elle soit directe ou indirecte, offre aux visiteurs les moyens d'appréhender son point de vue issu d'un travail créatif significatif d'une autre approche des œuvres anciennes. La programmation culturelle du musée, par l'intermédiaire de son auditorium ou de son service culturel, offre plusieurs possibilités pour assurer la diffusion du processus créatif d'un artiste vivant et les réflexions sous-jacentes à la création de son œuvre.

Le programme d'expositions temporaires d'œuvres contemporaines au Louvre, qui a connu sa conclusion avec l'exposition Mark Lewis en janvier 2015, visait incontestablement l'inscription d'un événement dans le parcours de visite et l'émergence d'un nouveau motif de visite. Mais il n'en demeure pas moins que le Louvre voulait assurer l'émergence d'un regard artistique sur ses collections dans l'espace des collections permanentes. En soumettant le regard du visiteur à des questions qui touchent l'activité créatrice à proprement parler, par l'intermédiaire des propositions plastiques d'artistes largement reconnus, la direction du Louvre de l'époque cherchait à redonner aux œuvres anciennes qu'il expose une actualité perdue et à assurer, dans l'enceinte du musée, un point de vue artistique à côté de celui historiciste proposé par l'accrochage. Comme le soutenait toujours Bernadac, «les artistes nous montrent le lien qui existe entre le passé et le présent, ce qui permet de redécouvrir les collections, de réactiver notre regard. Ils amènent avec eux leur regard artistique. Leur vision est enrichissante et stimulante. Leur contribution s'ajoute à celle des historiens et des scientifiques ${ }^{21}$. » Par le principe de la commande d'œuvres spécifiques, il s'agissait alors de donner à voir et à examiner ses collections par le prisme du travail de recherche de l'artiste d'aujourd'hui.

Il est nécessaire de souligner en conclusion que cette politique a été présentée comme engagement nécessaire du Louvre vis-à-vis de l'art vivant, " cette cause que l'on veut défendre », comme devait le rappeler

20 Lors de l'émission radiophonique de France-Culture, Tout arrive, du 20 avril 2007, consacrée à l'exposition Contrepoint 3 - De la Sculpture au Louvre, avec Gloria Friedmann, Arnaud Laporte, Annette Messager, Éric Troncy et Michel Verjux.
21 DOUAIRE, Pierre-Évariste. «Interviews, Marie-Laure Bernadac ». Paris-Art, $1^{\text {er }}$ juin 2010. <http://www.paris-art. com/interview-artiste/marie-laure-bernadac/bernadac-marie-laure/376.html> (consulté le 10 mars 2011). 
sa responsable lors du colloque « Exposer l'art contemporain dans les monuments historiques ${ }^{22}$. La direction de l'époque soutenait, en effet, qu'il en allait de sa responsabilité d'œuvrer en faveur de l'art contemporain, car " un musée dont les conservateurs, dont le directeur ne s'intéresse pas à l'art contemporain est un musée qui meurt ${ }^{23}$. Le désintéressement du musée à l'égard de l'art contemporain lui apparaissait ainsi comme un manque, une « infirmité en quelque sorte, qui est très dommageable ", car constitutif de la mortification des œuvres exposées en permanence et d'une incompréhension du sens de la création artistique. Aujourd'hui, les réflexions des artistes issues de leur confrontation sur les œuvres du Louvre sont en partie intelligibles grâce à une série de catalogues, d'articles, d'enregistrements aussi. Ces moyens de diffusion de l'information, même si celle-ci n'est que partielle lorsqu'il s'agit de processus créatif, s'inscrivent dans cet engagement, cette volonté de la direction du Louvre de faire découvrir à son public l'art de son temps, comme celle de faire découvrir l'art ancien au public de l'art contemporain. 